\title{
Watershed analysis of the target pulmonary artery for real-time localization of non-palpable pulmonary nodules
}

\author{
Xiang-Peng Chu ${ }^{1,2}$, Zi-Hao Chen ${ }^{1,2}$, Shao-Min Lin ${ }^{2}$, Jia-Tao Zhang ${ }^{2}$, Zhao-Wen Qiu ${ }^{3}$, Wen-Fang Tang ${ }^{4}$, \\ Rui Fu' ${ }^{2}$, Zhen-Bin Qiu ${ }^{2}$, Xue-Ning Yang ${ }^{2}$, Yi-Long Wu ${ }^{2}$, Qiang Nie ${ }^{2}$, Wen-Zhao Zhong ${ }^{1,2}$ \\ ${ }^{1}$ School of Medicine, South China University of Technology, Guangzhou, China; ${ }^{2}$ Guangdong Lung Cancer Institute, Guangdong Provincial Key \\ Laboratory of Translational Medicine in Lung Cancer, Guangdong Provincial People's Hospital \& Guangdong Academy of Medical Sciences, \\ Guangzhou, China; ${ }^{3}$ Tuomeng Technology Co. Ltd., Harbin, China; ${ }^{4}$ Department of Cardiothoracic Surgery, Zhongshan People's Hospital, \\ Zhongshan, China \\ Contributions: (I) Conception and design: XP Chu, Q Nie, WZ Zhong; (II) Administrative support: XN Yang, YL Wu; (III) Provision of study \\ materials or patients: ZH Chen, SM Lin; (IV) Collection and assembly of data: JT Zhang, R Fu; (V) Data analysis and interpretation: ZW Qiu, WF \\ Tang, ZB Qiu; (VI) Manuscript writing: All authors; (VII) Final approval of manuscript: All authors. \\ Correspondence to: Wen-Zhao Zhong; Qiang Nie. Guangdong Lung Cancer Institute, Guangdong Provincial Key Laboratory of Translational \\ Medicine in Lung Cancer, Guangdong Provincial People's Hospital \& Guangdong Academy of Medical Sciences, 106 Zhongshan Er Rd, Guangzhou \\ 510080, China. Email: syzhongwenzhao@scut.edu.cn; bulaier6480@163.com.
}

Background: Some pulmonary nodules are not suitable for computed tomography-guided percutaneous localization. This study aimed to investigate the feasibility and safety of real-time localization for these nonpalpable pulmonary nodules using watershed analysis of the target pulmonary artery during thoracoscopic wedge resection.

Methods: Watershed analysis is a novel technique that can be used to create a specific area on the lung surface for nodule localization. This analysis is performed by temporarily blocking the target pulmonary artery and using indocyanine green fluorescence during surgery. In our study, the surgery was simulated and evaluated preoperatively using a high-precision three-dimensional reconstruction model obtained by multidetector spiral computed tomography. The lung was observed using an infrared thoracoscopy system after an intravenous injection of indocyanine green $(2.5 \mathrm{mg} / \mathrm{mL})$, and the white-to-blue transitional zone was marked using electrocautery, after which a wedge resection was performed.

Results: A total of 25 out of 26 patients underwent successful wedge resection. The mean tumor size and depth based on computed tomography scans were $13.2 \pm 6.4$ and $12.2 \pm 7.8 \mathrm{~mm}$, respectively. The mean operation duration was $142.6 \pm 52.8 \mathrm{~min}$. The mean bleeding volume during surgery was $12.9 \pm 9.7 \mathrm{~mL}$. The mean drainage tube indwelling time was $35.6 \pm 20.0 \mathrm{~h}$, and the median length of postoperative stay was 3 days (range, 2-6 days).

Conclusions: Our experience showed that the watershed analysis of the target pulmonary artery for nodule localization was safe and feasible. It may become an effective and attractive alternative method for localizing non-palpable pulmonary nodules in selected patients undergoing thoracoscopic wedge resection.

Keywords: Localization; pulmonary nodule; thoracoscopic wedge resection; three-dimensional reconstruction

Submitted Dec 20, 2020. Accepted for publication Feb 26, 2021.

doi: $10.21037 /$ tlcr-20-1281

View this article at: http://dx.doi.org/10.21037/tlcr-20-1281 


\section{Introduction}

Lung cancer screening test results in the United States suggest that low-dose computed tomography (CT) screening in the high-risk lung cancer population not only increases the detection rate for early lung cancer but also reduces the disease-specific mortality rate by $20 \%$ (1).

With the current wide use of chest CT, more pulmonary nodules are being identified and confirmed using CT examination; most small pulmonary nodules are referred to as very early-stage lung cancer and are eligible for surgical resection $(2,3)$. The 5 -year survival rate in patients with pathologically confirmed atypical adenomatous hyperplasia, adenocarcinoma in situ (AIS), and minimally invasive adenocarcinoma (MIA) is almost $100 \%$ (4). Sublobar resection is considered an optimal and optional choice for these small pulmonary nodules (5-7).

Given the rapid progress of minimally invasive surgery, the early detection of pulmonary nodules might lead to fast-track surgery with less pain, faster recovery, and better quality of life $(8,9)$. At the same time, accurately locating pulmonary nodules is the key to minimally invasive surgery and for avoiding extended resection or thoracotomy (10).

Many localization techniques have been reported in previous studies; these include CT-guided hook-wire localization and methylene blue or indocyanine green (ICG) injection (11-13). Several studies have revealed extraordinary results when applying CT-guided percutaneous ICG localization techniques to localize pulmonary nodules $(14,15)$. However, there are always some pulmonary nodules that are not suitable for CT-guided localization and are a part of the "exclusion zone" of CT-guided percutaneous localization. These are outlined below:

(I) Some nodules are often located close to the mediastinum and far from the chest wall: for these nodules, the incidence of puncture-related complications is significantly high (16).

(II) Some tumors are close to the great vessels of the heart. Although it is technically feasible, puncture may lead to fatal massive hemorrhage; therefore, CT-guided puncture localization is not recommended.

(III) In few cases, the puncture route is obstructed by the scapula, rib, or other barriers, which may lead to the failure of CT-guided localization.

(IV) Some nodules are located under the pleura of pulmonary fissures, which are invisible and nonpalpable on the pleural surface. For these nodules, CT-guided localization is extremely difficult because of the high incidence of hook-wire unlooked or dye diffusion.

Segmentectomy and lobectomy have often been adopted to tackle this problem. However, pulmonary segmentectomy is a complicated operation requiring the severance of the pulmonary arteries, veins, and bronchi, which takes a long time. In addition, lobectomy for ground-glass nodules always results in the excessive loss of lung function (16). Therefore, we hope that through the improvement of surgical methods, these patients can also enjoy the convenience brought by wedge resection.

This study aimed to develop a strategy that allows the localization of non-palpable pulmonary nodules not suitable for CT-guided needle localization. To this end, we evaluated the safety and feasibility of the intraoperative real-time localization of non-palpable pulmonary nodules using a new method-the watershed analysis of the target pulmonary artery-in selected patients. We present the following article in accordance with the STROBE reporting checklist (available at http://dx.doi.org/10.21037/tlcr-20-1281).

\section{Methods}

\section{Patients}

A total of 26 patients were enrolled in this study between October 2019 and August 2020 at Guangdong Provincial People's Hospital. At our institution, the decision to use the watershed analysis of the target pulmonary artery for tumor localization is selective and depends on tumor parameters. We chose to localize lesions based on their sizes (favored for small lesions $<2 \mathrm{~cm}$ ), depth (depth of nodule from surface $<3 \mathrm{~cm}$ ), and morphology (ground-glass opacity). All patients were planning to undergo wedge resection for tumors and had been checked for allergies to ICG. Informed consent was obtained from all patients after the risks and benefits of the proposed surgery were explained to them by their surgeons. The study was conducted in accordance with the Declaration of Helsinki (as revised in 2013). The ethics committee of Guangdong Provincial People's Hospital approved the study (No. GDREC2019522H).

\section{Preoperative evaluation}

All patients underwent a preoperative workup using a 64 channel multidetector CT scan, performed in accordance with our center's standardized procedure.

Data from CT scans containing 1.0-mm slices were used to generate three-dimensional (3D)-CT images using a 

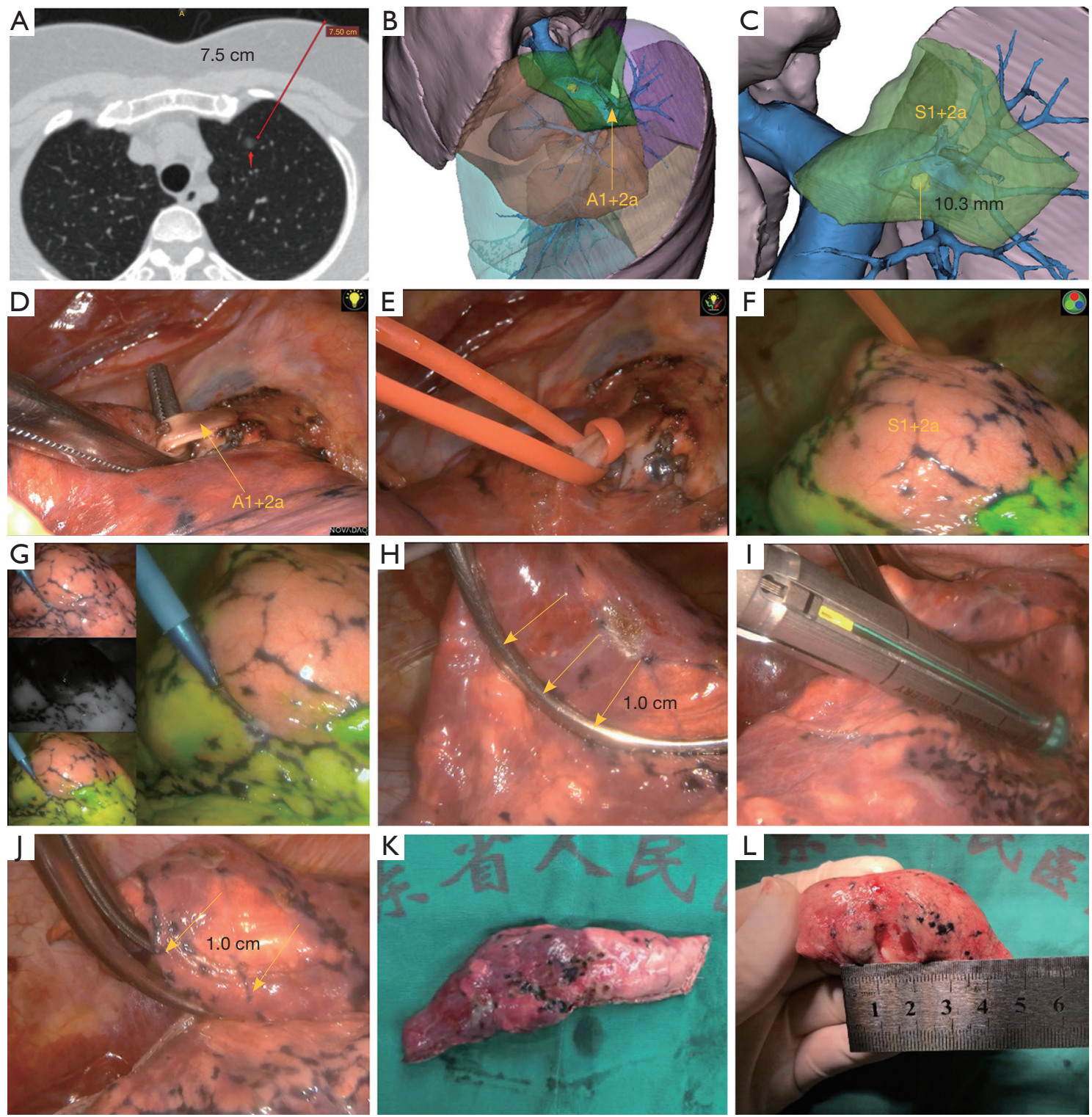

Figure 1 Localizing the nodule within the zone of negative staining using watershed analysis. (A) Preoperative computed tomography scan shows a pure ground-glass nodule (red arrow) located in the right upper lobe, about $7.5 \mathrm{~cm}$ from the lateral chest wall; (B) watershed analysis of segmental/subsegmental pulmonary arteries around the nodule was performed; (C) measuring the shortest distance between the lesion and the virtual cutting edge; (D) identifying the target artery intraoperatively; (E) the colored ribbon was used to ligate the target subsegmental artery with a slipknot; (F,G) Observation of the lung using an infrared thoracoscopy system after an intravenous injection of indocyanine green $(2.5 \mathrm{mg} / \mathrm{mL})$ and marking of the white-to-blue transitional zone by electrocautery; (H,I,J) to keep the margin at least $1 \mathrm{~cm}$ from the tumor, we performed an extended resection with a distance of $1.0 \mathrm{~cm}$ between the cutting edge and the marking; (K,L) wedge resection was performed successfully, and the lesion was confirmed.

3D reconstruction software (Diagnostic image processing software, TM-MIS1.0, Harbin, China), which was in turn used to evaluate the location of lesions and anatomy of the pulmonary arteries (Figure 1A).

At the same time, the function of watershed analysis in this software is used to simulate the negative staining region on the lung surface after the peripheral intravenous injection of ICG after blocking the target pulmonary artery.

First, watershed analysis of pulmonary segmental arteries around the nodule was performed (Figure 1B). 
After determining the pulmonary segment where the nodules are located, the anatomical characteristics of the pulmonary segmental artery should be used to determine whether further watershed analysis of subsegmental artery should be conducted. If the nodule was within the virtual watershed of the target pulmonary artery, we determined the position of the nodule in the target region and measured the distance between the lesion and the intersegmental/ intersubsegmental plane to guide the direction and range of the intraoperative surgical margin (Figure 1C).

It should be noted that the target artery to be blocked intraoperatively could be either segmental artery or subsegmental artery, depending on the specific location of pulmonary nodules and the anatomical characteristics of the target artery.

\section{Surgical procedure}

This was a pilot study, and it was important to assess the clinical feasibility of this new technique to verify that temporarily blocking the target subsegmental artery and using ICG fluorescence labeling (BTA-ICG) during surgery could allow the precise localization of nodules. We used the 3-4 cm muscle sparing anterolateral surgery approach under general anesthesia during single-lung ventilation, which was our standard procedure for thoracoscopic wedge resection. We performed video-assisted thoracoscopic surgery with double monitor guidance: one thoracoscopic television monitor and one $3 \mathrm{D}$ imaging system monitor were used. Intraoperative findings of the target pulmonary artery branching pattern were compared with the $3 \mathrm{D}-\mathrm{CT}$ pulmonary angiography images carefully, and the colored ribbon was used to ligate the target subsegmental artery with a slipknot (Figure 1D,E).

ICG $(5 \mathrm{~mL})$ was rapidly injected into a peripheral vein to observe the lung under infrared light. When illuminated with the infrared light, the lung clearly appeared to be separated into two areas, white and blue, according to the blood flow on the monitor. The transition zone between these two-colored areas was then marked on the visceral pleura using electrocautery (Figure 1F,G). Wedge resection was performed with staplers based on the preoperative evaluation of the $3 \mathrm{D}$ reconstruction and marking on the visceral pleura. If the tumor margin was not secured during the operation, we performed an extended resection regardless of the marking. We tried to keep the margin at least $1 \mathrm{~cm}$ from the tumor (Figure 1H,I,F,K,L).

Postoperative factors included pneumothorax and pleural effusion. Pneumothorax was defined as the apex (top) of the lung (a line) surpassing $3 \mathrm{~cm}$ and was diagnosed using chest roentgenography. Pleural effusion was defined by the blunting of the costophrenic angle on the chest roentgenogram.

\section{Statistical analysis}

The study variables were summarized using descriptive statistics. Continuous data are given as mean (SD) and median (interquartile ranges), whereas categorical variables are expressed as counts (percentages). Demographic data (age, sex), lesion characteristics (size, number, and location of nodules), information on the surgical approach (blocking target artery, wedge resection), length of postoperative hospital stay and complications were extracted from clinical records. Data analysis was conducting using the Statistical Package for Social Sciences (SPSS) (version 24, IBM Corp, Armonk, NY).

\section{Results}

Between October 2019 and August 2020, 26 consecutive patients who were scheduled for nodule localization and wedge resection were enrolled. The patient characteristics and surgical information for wedge resection are summarized in Table 1.

Overall, 25 out of 26 patients underwent successful intraoperative tumor localization and wedge resection. In one patient, localization was unsuccessful because the negative staining boundary was extremely unclear. Therefore, in this patient, segmentectomy was performed to completely remove the pulmonary nodule using the deflation/inflation method (17).

Nine male and 17 female patients were enrolled, with a median age of 54 years (range, 44-72 years). Most patients presented with solitary pulmonary nodules, but two patients had primary and secondary lesions in the lungs. However, we focused on the localization of their secondary lesions through BTA-ICG. On radiological examination, 13 pure ground-glass nodules, 10 partially solid nodules, two small solid nodules, and one cystic nodule were observed. The mean tumor size based on the CT scan was $13.2 \pm 6.4 \mathrm{~mm}$. The mean depth of the nodules from the surface was $12.2 \pm 7.8 \mathrm{~mm}$. No suspected lymph node metastasis was observed before surgery. All patients who underwent BTAICG localization showed no ICG-related adverse effects. The mean operation duration was $142.6 \pm 52.8 \mathrm{~min}$. The 
Table 1 Patient characteristics and tumor parameters

\begin{tabular}{|c|c|}
\hline Characteristics & Outcome \\
\hline \multicolumn{2}{|l|}{ Gender } \\
\hline Male & $9(34.6 \%)$ \\
\hline Female & $17(65.4 \%)$ \\
\hline Median age [range], years & $54[44-72]$ \\
\hline \multicolumn{2}{|l|}{ ASA class } \\
\hline 1 & $12(46.2 \%)$ \\
\hline II & $14(53.8 \%)$ \\
\hline Mean nodule size, $\mathrm{mm}$ & $13.2 \pm 6.4$ \\
\hline Mean depth of nodule from surface, $\mathrm{mm}$ & $12.2 \pm 7.8$ \\
\hline \multicolumn{2}{|l|}{ Nodule number } \\
\hline Solitary & $24(92.3 \%)$ \\
\hline Multiple & $2(7.7 \%)$ \\
\hline \multicolumn{2}{|l|}{ Nodule location } \\
\hline LUL & $12(46.0 \%)$ \\
\hline LLL & $2(7.7 \%)$ \\
\hline RUL & $8(30.8 \%)$ \\
\hline RLL & $4(15.4 \%)$ \\
\hline \multicolumn{2}{|l|}{ Radiological pattern } \\
\hline Pure ground glass nodule & $13(50.0 \%)$ \\
\hline Mixed ground glass nodule & $10(38.5 \%)$ \\
\hline Solid nodule & $2(7.7 \%)$ \\
\hline Cystic nodule & $1(3.9 \%)$ \\
\hline \multicolumn{2}{|l|}{ Pathological diagnosis } \\
\hline Adenocarcinoma in situ & $3(11.5 \%)$ \\
\hline Minimally invasive adenocarcinoma & $9(34.6 \%)$ \\
\hline Lepidic predominant adenocarcinoma & $1(3.9 \%)$ \\
\hline Acinar predominant adenocarcinoma & $9(34.6 \%)$ \\
\hline Benign disease & $4(15.4 \%)$ \\
\hline Mean Operation duration, min & $142.6 \pm 52.8$ \\
\hline Mean bleeding volume, $\mathrm{mL}$ & $12.9 \pm 9.7$ \\
\hline Median postoperative stay [range], d & $3[2-6]$ \\
\hline Mean drainage tube indwelling time, $\mathrm{h}$ & $35.6 \pm 20.0$ \\
\hline Pneumothorax (a line $\geq 3 \mathrm{~cm}$ ) (postoperative day 1 ) & $7(26.9 \%)$ \\
\hline Pleural effusion (postoperative day 1 ) & $7(26.9 \%)$ \\
\hline
\end{tabular}

RUL, right upper lobe; RLL, right lower lobe; LUL, left upper lobe; LLL, left lower lobe; ASA, American Society of Anesthesiologists. mean bleeding volume during surgery was $12.9 \pm 9.7 \mathrm{~mL}$. The incidence rates of pneumothorax and pleural effusion on postoperative day 1 were both $26.9 \%(7 / 26)$. The mean drainage tube indwelling time was $35.6 \pm 20.0 \mathrm{~h}$, and the chest tube was safely removed by the doctor when the patient met the discharge criteria. The median length of postoperative stay was 3 days (range, 2-6 days).

The pathological diagnoses of the 24 patients with solitary pulmonary nodules included 20 adenocarcinomas (76.9\%) and 4 benign diseases (15.4\%). Among the 20 adenocarcinomas, 2 were AIS, 8 were MIAs, 1 was lepidic predominant adenocarcinoma (LPA), and 9 were acinar predominant adenocarcinomas (APAs). Despite the pathological diagnosis of invasive adenocarcinomas (LPA and APA), we did not perform extensive resection since it has been reported that ground-glass opacity (GGO)-predominant nodules also have a good prognosis after sublobectomy according to JCOG0804/WJOG4507L Study (18).

In one of the two other patients with multiple pulmonary nodules, there was a pure ground-glass opacity (pGGO) in $\mathrm{S} 1+2 \mathrm{a}$ of the apicoposterior segment of the left upper lung, and a mixed GGO (mGGO) in S4a of the lingual segment. The pGGO in $\mathrm{S} 1+2 \mathrm{a}$ was less than $2.0 \mathrm{~cm}$ from the lung tip, and a wedge resection was performed directly; the tumor was pathologically confirmed as MIA. The mGGO in S4a was confirmed as benign by wedge resection after blocking the subsegmental artery of A4a and using ICG fluorescence. In another patient, there was a partial solid lesion with the size of $2.6 \mathrm{~cm} \times 1.5 \mathrm{~cm}$ in the apex of right upper lobe. Pulmonary lobectomy was performed immediately, after which the tumor was pathologically confirmed as invasive adenocarcinoma. A pGGO appeared in the anterior basal segment of the right lower lobe (approximate size of $8.0 \mathrm{~mm}$ $\times 9.0 \mathrm{~mm}$ ) (Figure 2A). On preoperative evaluation using a $3 \mathrm{D}$ reconstruction model, it appeared at the negative staining boundary after blocking A8b, at distance of approximately $4 \mathrm{~cm}$ from the bifurcation of A8b and $\mathrm{A} 7$ (Figure 2B,C,D). During the operation, A8b was temporarily blocked to form a negative staining boundary. At the same time, a $4-\mathrm{cm}$ measuring tube was used to determine the exact location of the nodule at the negative staining boundary, which was marked using electrocautery (Figure 2E,F,G,H,I). After wedge resection, the tumor was pathologically confirmed as AIS (Figure 27,K,L). Pathological biopsies of the lymph nodes all showed negative results. This case proved that the watershed analysis could not only help in accurately locating the nodules within the zone of negative staining but also in locating the nodules along the negative staining boundary, indicating that 

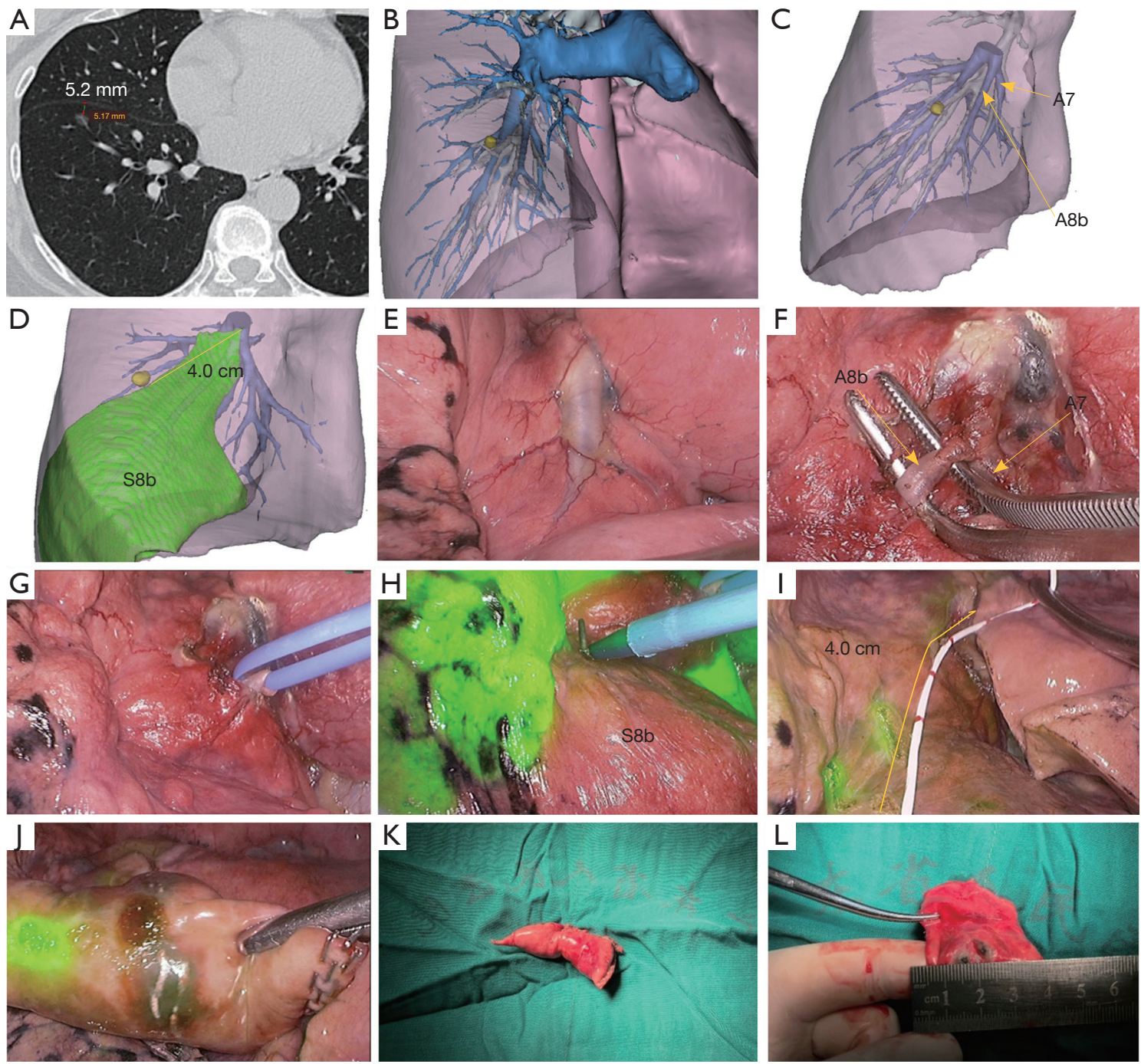

Figure 2 Localizing the nodule on the boundary of negative staining using watershed analysis. (A) Preoperative computed tomography scan shows a pure ground-glass nodule that appeared in the anterior basal segment of the right lower lobe with the depth of $5.2 \mathrm{~mm}$ from the pleura; (B,C) three-dimensional reconstruction model of the lesion and anatomy of the target subsegmental artery; (D) the lesion was located on the negative staining boundary of the target area with a distance of about $4 \mathrm{~cm}$ from the bifurcation of A8b and A7; (E,F) identifying the target artery intraoperatively; $(\mathrm{G})$ the colored ribbon was used to ligate the target subsegmental artery with a slipknot; (H) Observation of the lung using an infrared thoracoscopy system after an intravenous injection of indocyanine green $(2.5 \mathrm{mg} / \mathrm{mL})$ and marking of the whiteto-blue transitional zone by electrocautery; (I) the 4-cm measuring tube was used to determine the exact location of the nodule on the negative staining boundary; (J,K,L) wedge resection was performed, and the lesion was confirmed.

the application of this method can be further expanded.

\section{Conclusions}

In this study, we have implemented a safe and feasible method of watershed analysis of target pulmonary artery for the real-time localization of non-palpable pulmonary nodules, which are not suitable for traditional localization techniques.

By accurately simulating the positional relationship between the nodule and the virtual watershed before surgery, intraoperative BTA-ICG allowed for the formation of a clear negative staining boundary at the lung surface, which provided additional marker information, such as 
Table 2 Comparison of advantages and disadvantages among the watershed analysis, CT-guided localization and segmentectomy

\begin{tabular}{|c|c|c|}
\hline Localization methods & Advantages & Disadvantages \\
\hline Segmentectomy & $\begin{array}{l}\text { Resecting the nodules with the depth } \\
\text { of more than } 3.0 \mathrm{~cm} \text {, which are not } \\
\text { suitable for wedge resection }\end{array}$ & $\begin{array}{l}\text { (I) More sophisticated surgical techniques are needed; (II) longer duration } \\
\text { of operation; (III) removal of deeper and more lung parenchyma may } \\
\text { cause more complications }\end{array}$ \\
\hline
\end{tabular}

circles and lines, to improve the accuracy of localization, compared with points created by percutaneous CT-guided localization. One of the advantages of our method is that the position of the blood vessels to the nodules is fixed regardless of whether the lung is inflated or deflated. As long as the pulmonary nodules are within the blood supply, the nodules can be accurately located by blocking the target artery, even though the lung is deflated intraoperatively.

Our method is essentially a wedge resection, but the operation is similar to a simplified version of segmentectomy. We do not need to expose the segmental bronchus and veins, but only the target subsegmental arteries. At the same time, we do not need to use the cutting stapler to cut off the target artery. We only need to use the colored ribbon to ligate the target artery with a slipknot, so it is easier and safer.

Our method can also be applied to some nodules that can also be localized by CT-guided localization to relieve the pressure and save the resources of the CT rooms, as was done for the first patient in our study who had multiple nodules. At the same time, it can prevent the stress, anxiety, pain, pneumothorax, pulmonary hemorrhage, and other complications caused by preoperative CT-guided localization in these patients $(13,19)$.

ICG fluorescence labeling for the localization of small subpleural nodules has been reported previously (14). However, a dose of 0.1-0.2 mL ICG was delivered through a needle core and injected to localize a nearby lesion after local anesthesia and radiological localization. The limitation of their method was the longer time period between the staining of the nodule and the operation, which could cause the failure of CT-guided localization owing to dye diffusion. In contrast, our method allows intraoperative real-time localization, which could eliminate the problem of dye diffusion due to the long waiting time after localization.

In lung surgeries, thoracic adhesions are common. If severe extensive adhesions are present in the chest cavity, the application of CT-guided ICG injection would result in the failure of localization due to dye diffusion and dislodgement. However, our method can be used as a remedial measure to accurately localize nodules after adhesion isolation using electrocautery. The advantages and disadvantages of watershed analysis, traditional CT-guided localization, and segmentectomy are summarized in Table 2.

Currently, electromagnetic navigational bronchoscopy (ENB) using near-infrared fluorescence with ICG could also be used to localize pulmonary nodules located in the exclusion zone of CT-guided localization. Using ENB and ICG fluorescence for pulmonary tumor localization requires well-trained physicians and depends heavily on the equipment. In addition, it is difficult to localize nodules located in the upper lobes and at peripheral sites using bronchoscopy (20).

Despite the superiority of watershed analysis, there were some limitations in the present study. Fluoroscopy is necessary for tracing ICG fluorescence, and it is not extensively used in other hospitals. Owing to the presence of the traffic branches of the peripheral pulmonary arteries, occasionally, the negative staining boundary after blocking the target artery was not clear during our procedures, which could have influenced the surgeons' ability to locate the nodules. This problem could be detected and resolved by carefully analyzing the $3 \mathrm{D}$ reconstruction 
model preoperatively and performing segmentectomy intraoperatively.

In addition, to identify the target artery, we often required a slight separation of lung parenchyma surrounding the root of the target artery, which was associated with a certain risk of bleeding or alveolar air leaks and warranted better surgical skills. Although some patients showed signs of pneumothorax and pleural effusion on the chest roentgenogram on postoperative day 1 , the mean drainage tube indwelling time was just $35.6 \pm 20.0 \mathrm{~h}$, and the median length of postoperative hospitalization was 3 days (range, 2-6 days), which was comparable to the results of the routine chest tube drainage strategy after thoracoscopic wedge resection using CT-guided localization (21).

At the same time, this method requires a high accuracy for $3 \mathrm{D}$ reconstruction models and has the function of the watershed analysis of the pulmonary artery, which can be used to determine the locational relationship between nodules and target areas.

In summary, watershed analysis of the target pulmonary artery for real-time localization could provide surgeons performing precision sublobar resections with a timesaving surgical technique that delivers lower unnecessary intraoperative damage, especially for nodules located in the exclusion zone of CT-guided needle localization.

\section{Acknowledgments}

Funding: This work was supported by the National Natural Science Foundation of China (81673031, 81872510); Guangdong Provincial People's Hospital Young Talent Project (GDPPHYTP201902); High-level Hospital Construction Project (DFJH201801); Research Fund from Guangzhou Science and Technology Bureau (No. 201704020161); GDPH Scientific Research Funds for Leading Medical Talents and Distinguished Young Scholars in Guangdong Province (No.KJ012019449); Guangdong Basic and Applied Basic Research Foundation (No.2019B1515130002); and Guangdong Provincial Key Laboratory of Lung Cancer Translational Medicine (2017B030314120).

\section{Footnote}

Reporting Checklist: The authors have completed the STROBE reporting checklist. Available at http://dx.doi. org/10.21037/tlcr-20-1281
Data Sharing Statement: Available at http://dx.doi. org/10.21037/tlcr-20-1281

Conflicts of Interest: All authors have completed the ICMJE uniform disclosure form (available at http://dx.doi. org/10.21037/tlcr-20-1281). YLW serves as an unpaid Honorary Editor-in-Chief of Translational Lung Cancer Research from Jan 2015 to Jan 2021. WZZ serves as an unpaid Associate Editor-in-Chief of Translational Lung Cancer Research from Jan 2015 to Jan 2021. The other authors have no conflicts of interest to declare.

Ethical Statement: The authors are accountable for all aspects of the work in ensuring that questions related to the accuracy or integrity of any part of the work are appropriately investigated and resolved. The study was conducted in accordance with the Declaration of Helsinki (as revised in 2013). The study was approved by the ethics committee of Guangdong Provincial People's Hospital (No. GDREC2019522H) and informed consent was taken from all individual participants.

Open Access Statement: This is an Open Access article distributed in accordance with the Creative Commons Attribution-NonCommercial-NoDerivs 4.0 International License (CC BY-NC-ND 4.0), which permits the noncommercial replication and distribution of the article with the strict proviso that no changes or edits are made and the original work is properly cited (including links to both the formal publication through the relevant DOI and the license). See: https://creativecommons.org/licenses/by-nc-nd/4.0/.

\section{References}

1. Aberle DR, Adams AM, Berg CD, et al. Reduced lungcancer mortality with low-dose computed tomographic screening. N Engl J Med 2011;365:395-409.

2. Naidich DP, Bankier AA, MacMahon H, et al. Recommendations for the management of subsolid pulmonary nodules detected at CT: a statement from the Fleischner Society. Radiology 2013;266:304-17.

3. Suzuki K, Kusumoto M, Watanabe S, et al. Radiologic classification of small adenocarcinoma of the lung: radiologic-pathologic correlation and its prognostic impact. Ann Thorac Surg 2006;81:413-9.

4. Le Chevalier T. Adjuvant chemotherapy for resectable non-small-cell lung cancer: where is it going? Ann Oncol 
2010;21:vii196-8.

5. Nakamura H, Taniguchi Y, Miwa K, et al. Comparison of the surgical outcomes of thoracoscopic lobectomy, segmentectomy, and wedge resection for clinical stage I non-small cell lung cancer. Thorac Cardiovasc Surg 2011;59:137-41.

6. Carr SR, Schuchert MJ, Pennathur A, et al. Impact of tumor size on outcomes after anatomic lung resection for stage 1A non-small cell lung cancer based on the current staging system. J Thorac Cardiovasc Surg 2012;143:390-7.

7. Schuchert MJ, Abbas G, Awais O, et al. Anatomic segmentectomy for the solitary pulmonary nodule and early-stage lung cancer. Ann Thorac Surg 2012;93:1780-5.

8. Bendixen M, Jørgensen OD, Kronborg C, et al. Postoperative pain and quality of life after lobectomy via video-assisted thoracoscopic surgery or anterolateral thoracotomy for early stage lung cancer: a randomised controlled trial. Lancet Oncol 2016;17:836-44.

9. Vannucci F, Gonzalez-Rivas D. Is VATS lobectomy standard of care for operable non-small cell lung cancer? Lung Cancer 2016;100:114-9.

10. Khereba M, Ferraro P, Duranceau A, et al. Thoracoscopic localization of intraparenchymal pulmonary nodules using direct intracavitary thoracoscopic ultrasonography prevents conversion of VATS procedures to thoracotomy in selected patients. J Thorac Cardiovasc Surg 2012;144:1160-5.

11. Plunkett MB, Peterson MS, Landreneau RJ, et al. Peripheral pulmonary nodules: preoperative percutaneous needle localization with CT guidance. Radiology 1992;185:274-6.

12. Finley RJ, Mayo JR, Grant K, et al. Preoperative computed tomography-guided microcoil localization of small peripheral pulmonary nodules: a prospective randomized controlled trial. J Thorac Cardiovasc Surg 2015;149:26-31.

13. Zaman M, Bilal H, Woo CY, et al. In patients undergoing video-assisted thoracoscopic surgery excision, what is the best way to locate a subcentimetre solitary pulmonary nodule in order to achieve successful excision? Interact Cardiovasc Thorac Surg 2012;15:266-72.

14. Zhang C, Lin H, Fu R, et al. Application of indocyanine green fluorescence for precision sublobar resection. Thorac Cancer 2019;10:624-30.

15. Anayama T, Hirohashi K, Miyazaki R, et al. Near-infrared dye marking for thoracoscopic resection of small-sized pulmonary nodules: comparison of percutaneous and bronchoscopic injection techniques. J Cardiothorac Surg 2018;13:5.

16. Ciriaco P, Negri G, Puglisi A, et al. Video-assisted thoracoscopic surgery for pulmonary nodules: rationale for preoperative computed tomography-guided hookwire localization. Eur J Cardiothorac Surg 2004;25:429-33.

17. Scanagatta P, Sestini S, Sverzellati N. eComment. Pulmonary segmentectomies: should we follow segmental veins or deflation/inflation lines? Interact Cardiovasc Thorac Surg 2013;17:980-81.

18. Suzuki K, Watanabe SI, Wakabayashi M, et al. A singlearm study of sublobar resection for ground-glass opacity dominant peripheral lung cancer. J Thorac Cardiovasc Surg 2020. [Epub ahead of print]. doi: 10.1016/ j.jtcvs.2020.09.146.

19. Okada M, Mimura T, Ikegaki J, et al. A novel videoassisted anatomic segmentectomy technique: selective segmental inflation via bronchofiberoptic jet followed by cautery cutting. J Thorac Cardiovasc Surg 2007;133:753-8.

20. Ferrari-Light D, Geraci TC, Sasankan P, et al. The Utility of Near-Infrared Fluorescence and Indocyanine Green During Robotic Pulmonary Resection. Front Surg 2019;6:47.

21. Zhang JT, Tang YC, Lin JT, et al. Prophylactic airextraction strategy after thoracoscopic wedge resection. Thorac Cancer 2018;9:1406-12.
Cite this article as: Chu XP, Chen ZH, Lin SM, Zhang JT, Qiu ZW, Tang WF, Fu R, Qiu ZB, Yang XN, Wu YL, Nie Q, Zhong WZ. Watershed analysis of the target pulmonary artery for real-time localization of non-palpable pulmonary nodules. Transl Lung Cancer Res 2021;10(4):1711-1719. doi: 10.21037/ tlcr-20-1281 